\title{
Intensity and directionality of bat echolocation signals
}

\author{
Lasse Jakobsen ${ }^{1 *}$, Signe Brinkløv ${ }^{2}$ and Annemarie Surlykke ${ }^{1}$ \\ 1 Institute of Biology, University of Southern Denmark, Odense, Denmark \\ ${ }^{2}$ Department of Biology, Western University, London, ON, Canada
}

Edited by:

Cinthya F. Moss, University of

Maryland, USA

Reviewed by:

James A. Simmons, Brown

University, USA

Ben Falk, University of Maryland,

USA

\section{${ }^{*}$ Correspondence:}

Lasse Jakobsen, Institute of Biology,

University of Southern Denmark,

Campusvej 55, Odense $M$,

DK-5230, Denmark.

e-mail: lasse@biology.sdu.dk
The paper reviews current knowledge of intensity and directionality of bat echolocation signals. Recent studies have revealed that echolocating bats can be much louder than previously believed. Bats previously dubbed "whispering" can emit calls with source levels up to $110 \mathrm{~dB}$ SPL at $10 \mathrm{~cm}$ and the louder open space hunting bats have been recorded at above $135 \mathrm{~dB}$ SPL. This implies that maximum emitted intensities are generally $30 \mathrm{~dB}$ or more above initial estimates. Bats' dynamic control of acoustic features also includes the intensity and directionality of their sonar calls. Aerial hawking bats will increase signal directionality in the field along with intensity thus increasing sonar range. During the last phase of prey pursuit, vespertilionid bats broaden their echolocation beam considerably, probably to counter evasive maneuvers of eared prey. We highlight how multiple call parameters (frequency, duration, intensity, and directionality of echolocation signals) in unison define the search volume probed by bats and in turn how bats perceive their surroundings. Small changes to individual parameters can, in combination, drastically change the bat's perception, facilitating successful navigation and food acquisition across a vast range of ecological niches. To better understand the function of echolocation in the natural habitat it is critical to determine multiple acoustic features of the echolocation calls. The combined (interactive) effects, not only of frequency and time parameters, but also of intensity and directionality, define the bat's view of its acoustic scene.

Keywords: intensity, directionality, beam shape, bat, echolocation, biosonar

\section{INTRODUCTION}

The evolutionary success of bats is accredited to their ability, as the only mammals, to fly and navigate in darkness by echolocation, thus filling a niche exploited by few other predators. Over $90 \%$ of all bat species use echolocation to localize obstacles in their environment by comparing their own high frequency sound pulses with returning echoes (Griffin, 1958). The ability to localize and identify objects without the use of vision allows bats to forage for airborne nocturnal insects, but also for a diverse range of other food types including motionless perched prey or nonanimal food items (Schnitzler and Kalko, 2001; Brinkløv et al., 2011; Geipel et al., 2013).

The agility and precision with which bats navigate and forage in total darkness, is in large part due to the accuracy and flexibility of their echolocation system. The echolocation clicks of the few echolocating Pteropodidae (Rousettus) are fundamentally different from the echolocation sounds produced in the larynx that we focus on here, and thus not part of this review. Many studies have shown that bats adapt their echolocation calls to a variety of conditions, changing duration and bandwidth of each call and the rate at which calls are emitted in response to changing perceptual demands (Griffin et al., 1960; Schnitzler and Kalko, 2001). In recent years the intensity and directionality of echolocation signals has received increasing research attention and it is becoming evident that these parameters also play a major role in how bats successfully navigate and forage. To perceive an object in its surroundings, a bat must ensonify the object with enough energy to return an audible echo. Hence, the intensity and duration of the emitted signal act together to determine how far away a bat can echolocate an object. Equally important is signal directionality. Bat echolocation calls are directional, i.e., more call energy is focused in the forward direction than to the sides (Simmons, 1969; Shimozawa et al., 1974; Mogensen and Møhl, 1979; Hartley and Suthers, 1987, 1989; Henze and O'Neill, 1991). An object detectable at $2 \mathrm{~m}$ directly in front of the bat may not be detected if it is located at the same distance but off to the side. Consequently, at any given echolocation frequency and duration, it is the combination of signal intensity and signal directionality that defines the search volume, i.e., the volume in space where the bat can detect an object.

The aim of this review is to summarize current knowledge about intensity and directionality of bat echolocation calls, and show how both are adapted to habitat and behavioral context. Finally, we discuss the importance of active motor-control to dynamically adjust both signal intensity and directionality to solve the different tasks faced by echolocating bats.

\section{INTENSITY}

Call intensity is a main determinant of echolocation range, i.e., the distance from a bat where objects, such as obstacles and food, reflect echoes intense enough for detection. The more intense the call, the further sound travels from the bat and the larger the echolocation range. Emitted intensities (source level) of bat echolocation signals are referenced to a standard distance of 
$10 \mathrm{~cm}$ from the bat's mouth. Thus, when recording bats at a distance, one must add the transmission loss due to geometric spreading $\left[20 \times \log _{10}(\mathrm{R})\right]$ and frequency-dependent atmospheric attenuation (ANSI, 1995) over the distance from the bat to the microphone.

When Griffin first investigated how loud bats call, he found that insectivorous bats flying in open space, e.g., aerial hawking vespertilionids, called at around $110 \mathrm{~dB}$ SPL (Sound Pressure Level; re. $20 \mu \mathrm{Pa}$ at $0.1 \mathrm{~m}$ ) and closed-space gleaners operating in or near vegetation, like the phyllostomid Carollia perspicillata, called at around $70 \mathrm{~dB}$ SPL (Griffin, 1958). Consequently, Griffin divided bats into two groups, the loud insectivorous bats, and the "whispering" gleaning bats. Recordings from the field have since shown that bats are orders of magnitude louder than what Griffin measured, and the border between loud and whispering is much blurrier than initially believed. Open-space insectivorous bats emit calls up to, and beyond, $140 \mathrm{~dB}$ SPL (Holderied et al., 2005; Surlykke and Kalko, 2008). Remarkably, even "whispering" bats are capable of emitting calls up to $110 \mathrm{~dB}$ SPL (Brinkløv et al., 2009). This means that, while echolocation in air is still a relatively short-range system, its range is considerably larger than first assumed.

The huge difference between the values for signal intensity obtained by Griffin and more recent measurements illustrates the great flexibility of the echolocation system. Bats dynamically adjust signal intensity to changes in their environment and the task at hand, lowering the output as they approach objects such as prey or vegetation. The dynamic range, or the difference between the loudest and the quietest calls emitted by individual bats is in the order of at least $30-40 \mathrm{~dB}$ for most species. When object detection occurs at long range or under predictable lab conditions most studies report a reduction in output level of around $6 \mathrm{~dB}$ for every halving of distance to the target (Hartley, 1992b; Hiryu et al., 2007, 2008; Brinkløv et al., 2010; Koblitz et al., 2010, 2011; Nørum et al., 2012). If the object reflects impinging sound like a point target, the echo level at the bats ears would increase by $12 \mathrm{~dB}$ per halving of distance if the bat emitted a constant source level. Thus, the consequence would be an enormous increase in echo level through a pursuit, e.g., $+80 \mathrm{~dB}$ from detection at $5 \mathrm{~m}$ to capture at $5 \mathrm{~cm}$, likely to overload central auditory processing. The output reduction of $6 \mathrm{~dB}$ per halving of distance removes half of the echo increase such that the sound pressure at the bat's ear increases by only $6 \mathrm{~dB}$ per halving of distance. Further, psychophysical experiments have shown that sensitivity on the receiver side is not constant, but decreases by the remaining $6 \mathrm{~dB}$ for each halving of distance probably due to contraction of the bats middle-ear muscles (Suga and Jen, 1975). Hence, in a predictable situation the combined adjustment of output and input results in echoes perceived at a relatively stable intensity (Henson, 1965; Suga and Jen, 1975; Hartley, 1992a). Data from more unpredictable situations in the natural environment have also indicated a $6 \mathrm{~dB}$ reduction in signal output intensity per distance halved. New data, however, show that the reduction in intensity for individual approaches is mostly much steeper in the wild, up to as much as $30 \mathrm{~dB}$ per halving of distance, with considerable variation. The relatively shallow slopes reported from other field studies are probably the result of pooling multiple sequences

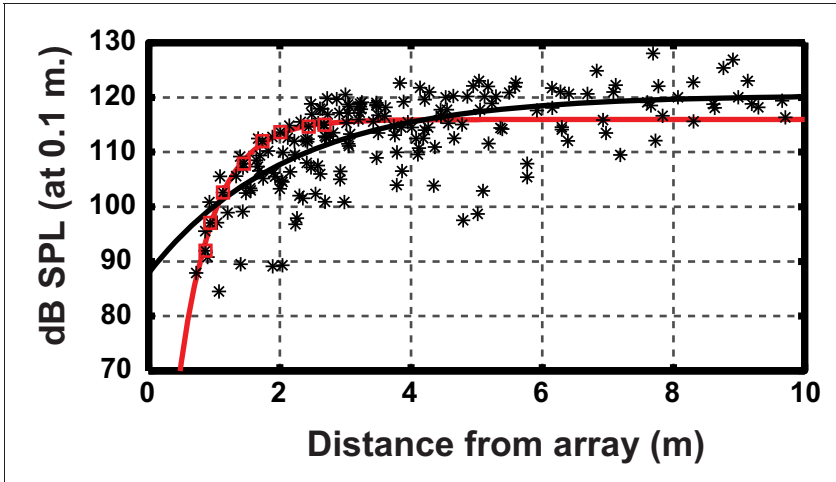

FIGURE 1 | Relationship between source level and distance to the microphone array for Myotis daubentonii. Datapoints (*) from 14 approaches. The black line is the least-squares fitted exponential function to the entire data set (for details see Nørum et al., 2012). Red squares highlight a single approach and the red line is the fitted function using only these points, showing a much steeper slope. The figure illustrates that estimating the slope using a large data set, comprising many individual steep slopes with different onsets, can yield an artificially shallow slope as compared to individual approaches. Data points from Nørum et al. (2012).

with steep slopes but initiated at different distances (Figure 1, Nørum et al., 2012). The results suggest that sudden detection of prey or obstacles at close range may prompt an initial dramatic intensity reduction. Curiously, for bats landing on an extended surface, the reduction in output intensity is likewise within the $6 \mathrm{~dB}$ per halving of distance range (Koblitz et al., 2011). If the sensitivity on the receiver side changes as well, this results in a gradual decrease in perceived echo strength as the bat approaches the surface.

There may be a less clear-cut separation between loud and whispering bats than previously assumed, but it is still evident that bats flying close to or within dense vegetation are considerably less intense than bats flying in open space. This is true for species that differ in overall habitat use, but also for individual bats switching between habitats with varying degrees of clutter. Under field conditions, the trawling insectivorous phyllostomid Macrophyllum macrophyllum lowers mean signal intensity from $111 \mathrm{~dB}$ SPL in open space to $105 \mathrm{~dB}$ SPL in semicluttered space. Signal intensity is further reduced to $100 \mathrm{~dB}$ SPL when $M$. macrophyllum navigates a small flight room, demonstrating an obvious dynamic adjustment of output intensity in response to varying degrees of habitat clutter (Brinkløv et al., 2010).

The adjustment of signal intensity in M. macrophyllum occurs in parallel with changes in signal duration and peak frequency. Open space calls are not only louder, but also longer and with lower peak frequency than those emitted in semi- or densely cluttered conditions. These changes all contribute to an increase in sonar range in open space. The increased duration increases the signal energy and the lower frequency reduces the effects of atmospheric attenuation. Attenuation of sound in air increases drastically with frequency (Lawrence and Simmons, 1982; ANSI, 1995) which presumably represents a major constraint for echolocating bats resulting in a trade-off between sonar range on one 
hand and resolution and localization on the other (Kalko and Schnitzler, 1993). The low intensities and high frequencies emitted by most gleaners in clutter likely indicate that sonar range is not an issue. Thus, the low intensities serve to prevent selfdeafening and the high frequencies serve to increase resolution and localization (Kalko and Schnitzler, 1993).

The use of high frequencies also increases clutter rejection along the acoustic axis when the prey is closer to the bat than the clutter. This is because the increased atmospheric attenuation at higher frequencies will generate a relatively weaker echo the further away an object is. An increase in emitted frequency from 45 to $90 \mathrm{kHz}$ increases atmospheric attenuation from 1.4 to $4 \mathrm{~dB} / \mathrm{m}$ (at $25^{\circ} \mathrm{C}$ and $80 \%$ humidity). If clutter is present $0.5 \mathrm{~m}$ behind the prey, the prey/clutter echo-ratio will be $2.6 \mathrm{~dB}$ higher at $90 \mathrm{kHz}$ than at $45 \mathrm{kHz}$, thus increasing prey conspicuousness.

An added advantage of using low intensity echolocation is that it may prevent prey from detecting an approaching bat. The sound pressure reaching the prey will always be higher than the echo returning to the bats ears, but eared insects, such as moths, generally have much higher hearing thresholds than bats (Wenstrup, 1984; Schmidt et al., 1992; Esser and Daucher, 1996; Koay et al., 1997; Surlykke et al., 1999). While intensity at the insect increases by $20 \times \log _{10}(\mathrm{R})$ as the bat approaches, the echo the bat receives increases by $40 \times \log _{10}(\mathrm{R})$. Thus, every time the bat halves the distance to the prey, the sound pressure increases with $6 \mathrm{~dB}$ at the prey and $12 \mathrm{~dB}$ at the bat. By concurrently reducing its output level by $6 \mathrm{~dB}$, the bat maintains a constant sound pressure at the prey, but still increases the returning echoes by $6 \mathrm{~dB}$. This keeps prey out of the loop while increasing echo strength for the approaching bat (Surlykke, 1988). By emitting low intensity calls, the aerial hawking bat, Barbastellus barbastellus, can detect its prey before the prey detects the bat, and by reducing its output level during approach it can remain undetected during the pursuit (Goerlitz et al., 2010). The low-intensity calls from $B$. barbastellus do come at a cost; a reduction in output level also reduces the detection distance for the bat, but given that $B$. barbastellus feeds almost exclusively on eared insects, the benefit of not being detected seems to outweigh the cost of operating at short range.

\section{DIRECTIONALITY}

A directional echolocation signal provides bats with a number of advantages over an omni-directional signal: (1) inherent directional information; by focusing sound in the forward direction, returning echoes are likely to originate from that direction, simplifying object localization. (2) A reduction in clutter; when less sound is radiated to the back and sides of the bat, less sound is reflected off objects of little or no interest in these directions, reducing the amount of information the bat has to process. (3) An increase in source level (on-axis intensity); by focusing energy in a narrow cone instead of radiating it in all directions. On the other hand, a highly directional sound beam will also restrict the bats "field of view" which may be a disadvantage in certain situations.

Beam shape is a spatial filter that determines what information is available to the bat and what information is filtered out before echoes return. This may be critical in light of the very short time bats have to make decisions. A typical pursuit often takes less than half a second and the time to process information and make decisions on a call-to-call basis is even shorter, perhaps a few tens of milliseconds. A clear advantage of a highly adapted and dynamic emission (and reception) system is a reduction in processing load on the receiving side i.e., smart sensing over smart processing. This may be one of the adaptations that allow for the very fast reaction times in echolocating bats, subsequently leading to high foraging, and, in turn, evolutionary, success.

Beam shape is determined by the size and shape of the sound emitter and the frequency of the emitted signal (Strother and Mogus, 1970; Urick, 1983) such that an increase in size or frequency generates a more directional sound beam (Figure 2). Frequency is easily measured, but emitter size and shape is not as apparent when dealing with live animals, let alone bats in flight. For bats emitting sound through the open mouth, gape size presumably dictates directionality. Opening the mouth more while emitting a given frequency will generate a more directional beam and vice versa (Surlykke et al., 2009). For nose emitting bats, beam shape is likely dictated by the distance between the nostrils and the size and shape of the nose-leaf (Hartley and Suthers, 1987).

Beam directionality has so far been measured in 17 bat species from seven different families (Figure 3). The methods differ substantially. Many studies were performed on restrained bats, often with calls elicited through stimulation of the brain by implanted electrodes (Shimozawa et al., 1974; Schnitzler and Grinnell, 1977; Mogensen and Møhl, 1979; Hartley and Suthers, 1987, 1989; Henze and O'Neill, 1991; Hiryu et al., 2006). This made it easy

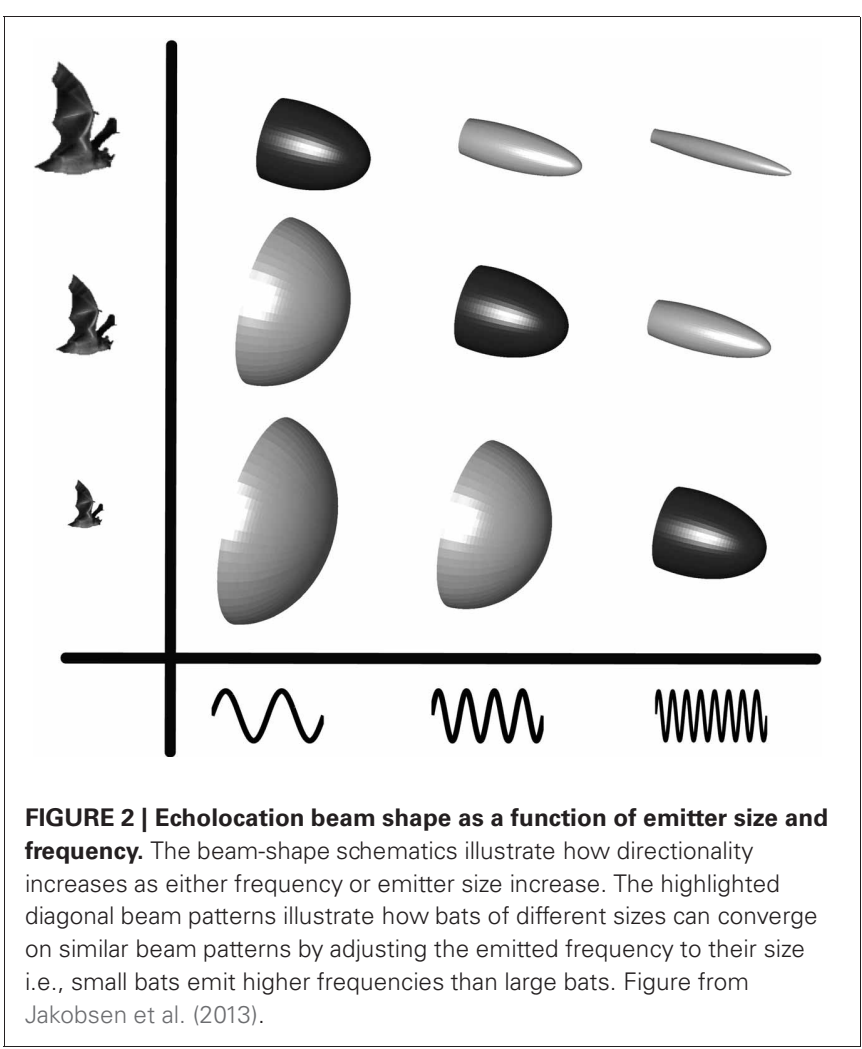


to control the bat's position and acoustic axis, but probably prevented active beam shape control by the bats, and in some cases produced calls very different from those produced by freely behaving bats. A few early and most later studies focus mostly on freely behaving bats in the lab (Griffin, 1958; Simmons, 1969; Ghose and Moss, 2003; Jakobsen and Surlykke, 2010; Jakobsen et al., 2012, 2013) with one report from the field (Surlykke et al., 2009). In spite of the large differences in methodology, a few general trends emerge from the data set. It is clear that all bats recorded emit directional signals and it is also clear that they emit a bilaterally symmetrical sound beam. Most results come from the Vespertilionidae (nine species), where directionality is remarkably uniform across species for bats echolocating under similar conditions, in spite of large differences in bat size and emitted frequency (Figure 3). This indicates that directionality may have been one of the major constraints on the evolution of echolocation frequency, forcing small bats to echolocate at higher frequencies to produce a sufficiently directional beam (Jakobsen et al., 2013). Echolocation frequency is also important for echo reflection (Møhl, 1988; Pye, 1993) and ranging accuracy (Stamper et al., 2009) and frequency-dependent directionality may help bats segregate target and clutter echoes (Bates et al., 2011). Thus, echolocation frequency is probably under several simultaneous evolutionary constraints. There is a negative correlation between size and echolocation frequency in most families of bats (Jones, 1999), and it will be interesting to see if this translates into a convergence of beam width for other families than the Vespertilionidae. Data from the emballonurids seem to deviate from this pattern. Cormura brevirostris emits a narrower beam in the flight cage than does Saccopteryx bilineata (DI of 11.5 and $9.3 \mathrm{~dB}$, respectively, Figure 3 ), but given that emission patterns from only two species have been recorded so far (Jakobsen et al., 2012), conclusive evidence is still lacking. In contrast to other bats Phyllostomids do not appear to show correlation between body size and emitted frequency. Curiously, data show that nose-leaf size is not correlated with body size either in phyllostomids (Hartley and Suthers, 1987; Jones, 1999). Even though the nose-leaf is not the emitter per se, it has been shown to define the vertical directionality (Hartley et al., 1989; Vanderelst et al., 2012). Thus, if directionality is a driving force for echolocation frequency in phyllostomids as well, we would expect to find that the emitted frequency is correlated to the size of the nose-leaf and the nostril separation but not to body size.

Bats adapt many features of their echolocation calls in response to changes in their surroundings and to behavioral context. Signal bandwidth and peak frequency are increased in many species when they navigate in cluttered space probably to improve resolution and localization accuracy (Kalko and Schnitzler, 1993). Further, an increase in frequency will result in an increase in directionality. However, as directionality depends on both frequency and effective emitter size, combined changes of the two may result in the opposite effect. This seems to be the case for

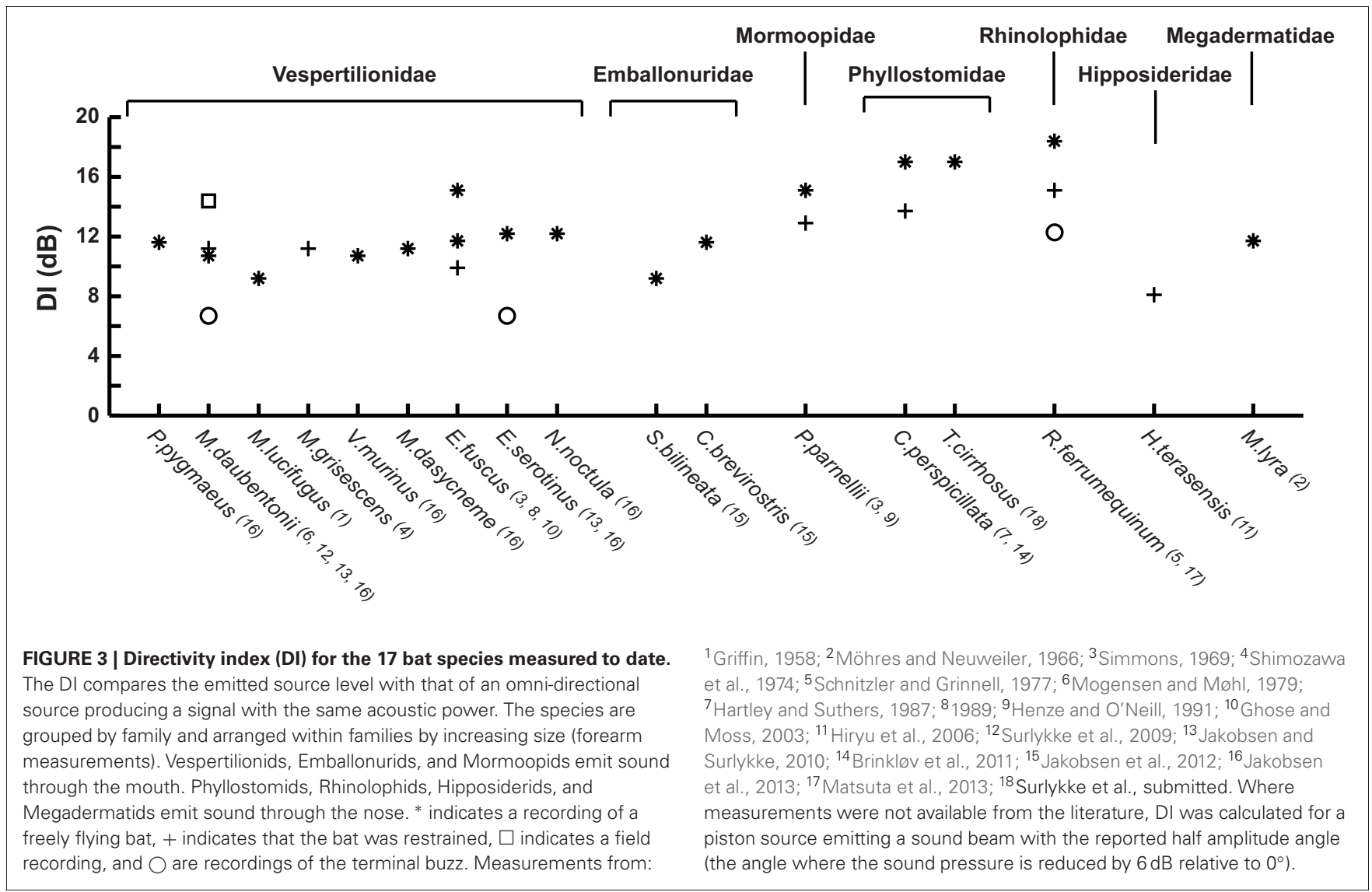


the vespertilionid Myotis daubentonii. When navigating in the field, M. daubentonii emits calls with lower peak frequency than in the lab ( 45 vs. $55 \mathrm{kHz}$ ), which by itself would produce a less directional beam. Yet, the signals are more directional in the field than in the lab, presumably because the bats also increase their emitter size by opening the mouth wider (Surlykke et al., 2009).

Echolocating bats must adjust directionality not only to adapt to the environment, but also in response to rapid changes in the perceived echo-scene, especially when hunting prey doing their best to escape. At least six orders of insects have ultrasound sensitive ears and exhibit "anti-bat tactics" i.e., they perform erratic escape behaviors like power dives and passive falls in response to intense ultrasound (Miller and Surlykke, 2001). The relatively high directionality of echolocation signals will, in close proximity to prey, become a disadvantage to the bat since the prey only has to move a short distance to escape the bat's sound beam. M. daubentonii and Eptesicus serotinus (Vespertilionidae) in fact broaden their beam in the last part of prey pursuit by lowering call frequency by roughly an octave (Jakobsen and Surlykke, 2010). A similar frequency drop is seen in a large number of insectivorous vespertilionids and is known as Buzz II (Figure 4). Buzz II calls have been thought an artifact of the extremely high repetition rate of calls emitted during this stage of pursuit, sometimes exceeding 200 calls/s (Kalko and Schnitzler, 1989; Faure and Barclay, 1994). From a purely physiological perspective, however, this seems unlikely as such fast call rates would result in additive tension build-up in the laryngeal muscles, ultimately increasing, rather than reducing call frequency during the buzz (Ratcliffe et al., 2013). Further, many species of echolocating bats use repetition rates as high as vespertilionids but without the frequency drop (Surlykke et al., 1993; Ibáñez et al., 2002).
Thus, we argue that the lower frequency of Buzz II calls is not a non-functional epiphenomenon. Rather, it is an adaptive feature that broadens the echolocation beam considerably in the last phase of pursuit to counter the evasive maneuvers performed by many eared insects when exposed to intense ultrasound (Jakobsen and Surlykke, 2010). This argument is corroborated by recent results from horseshoe bats. During prey pursuit, the Japanese greater horseshoe bat, Rhinolophus ferrumequinum nippon, will likewise broaden its echolocation beam, but only when the prey moves (Matsuta et al., 2013). In horseshoe bats beam broadening is, contrary to vespertilionids, not achieved by lowering the call frequency. The mechanism underlying the change in beam shape is still unknown, but it is likely facilitated by manipulating the fine structures of the nose leaf (Feng et al., 2012).

As discussed above, the optimal directionality is likely to differ from situation to situation, but also between bats with different feeding ecology. Gleaning bats, foraging in dense vegetation for inconspicuous stationary food items, presumably benefit more from a narrower beam shape than an open space aerial hawking bat. Directionality data from the frugivorous bat, C. perspicillata, and the frog-eating bat, Trachops cirrhosus, corroborate this. Both phyllostomid species have echolocation beam widths with $D I$ of $17 \mathrm{~dB}$ (half amplitude angle of $\sim 15^{\circ}$ ) when flying in a flight cage (Brinkløv et al., 2011, Surlykke et al., submitted). This is considerably narrower than the 10-12 $\mathrm{dB}$ (half amplitude angle of $\sim 37^{\circ}$ ) measured for aerial hawking bats flying in similar conditions (Ghose and Moss, 2003; Jakobsen et al., 2013) (Figure 3). So far the only report of active beam shape adjustments in noseemitting bats come from the horseshoe bats (Matsuta et al., 2013), an adjustment likely facilitated by manipulating the shape of the nose leaf. However, the phyllostomid, M. macrophyllum, shifts

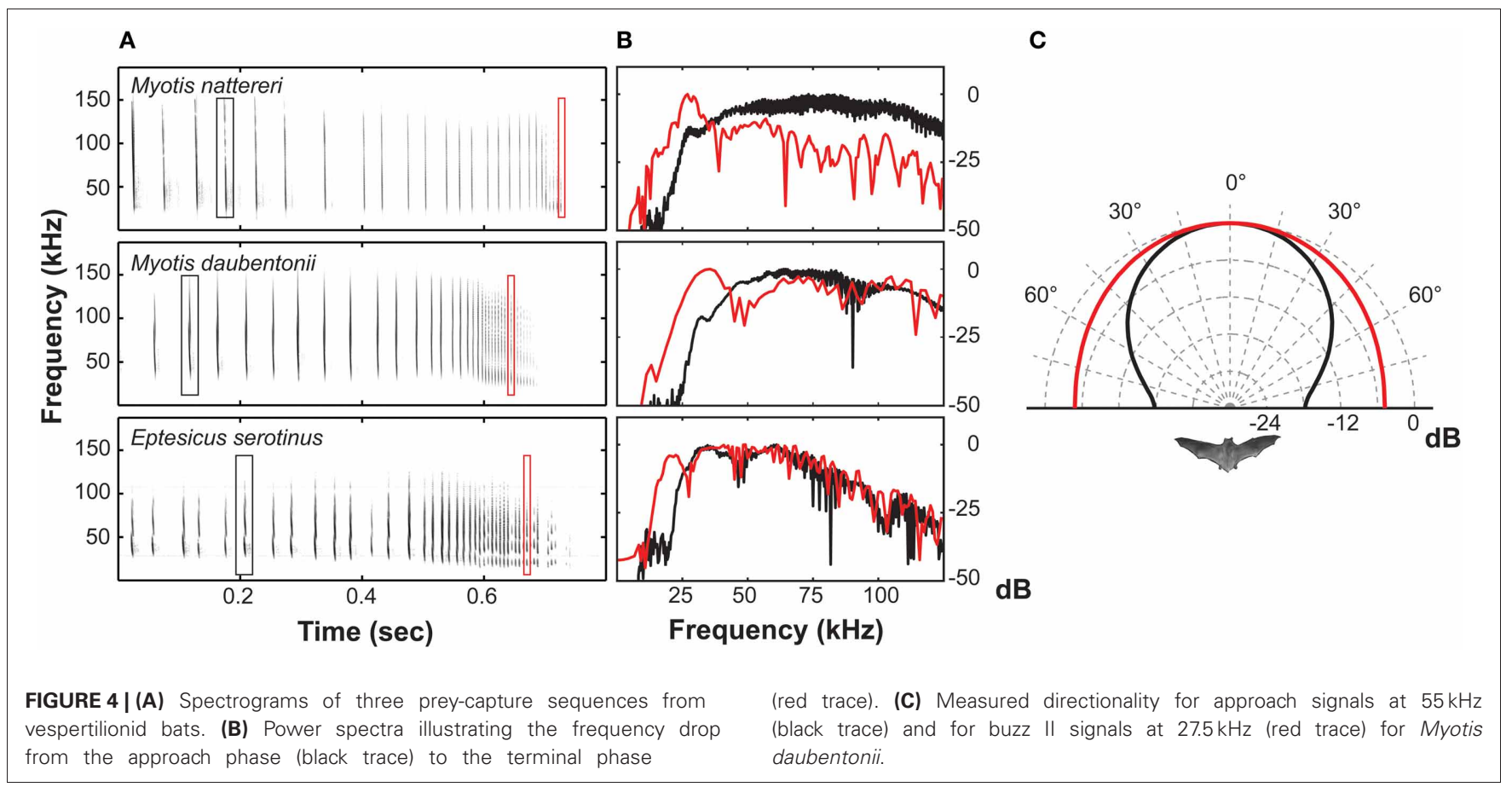


maximum energy to the second harmonic in the open, but to the third (or fourth) harmonic in cluttered space (Brinkløv et al., 2010), indicating that phyllostomid bats may also alter beam shape by changing emitted frequency.

\section{MODELING EMISSION PATTERNS}

Modeling bats as physical sound emitters allows predictions about parameters, which cannot or have not been measured. The simple piston model describes the beam pattern of a rigid circular piston oscillating in an infinite baffle and has been used as a model for the emission pattern of mouth emitting bats (Strother and Mogus, 1970; Mogensen and Møhl, 1979):

$$
R_{P}(\theta)=\left|\frac{2 \times J_{1}(k \times a \times \sin (\theta))}{k \times a \times \sin (\theta)}\right|
$$

$R_{p}(\theta)$ is the ratio between the on-axis pressure and the pressure at an angle $\theta, J_{1}$ is a first order Bessel function of the first kind, $k$, the wavenumber $=2 \pi / \lambda, \lambda$ the wavelength, and $a$ is the radius of the piston. Even though, there are a number of obvious differences between bats and the model, the model performs surprisingly well in predicting the emission pattern from mouth emitting bats (Mogensen and Møhl, 1979; Hartley and Suthers, 1989; Jakobsen and Surlykke, 2010; Jakobsen et al., 2012).

The emission pattern from nose emitting phyllostomid bats has been modeled using a two point-source model, but with limited success in particular for freely flying bats (Strother and Mogus, 1970; Hartley and Suthers, 1987; Brinkløv et al., 2011). A model simulating two small pistons with the same separation as the nostrils appears a much better approximation to the horizontal directionality of phyllostomid bats (Vanderelst et al., 2010), but the vertical pattern has so far not been successfully modeled by simple means (see Zhuang and Müller, 2006, 2007; Vanderelst et al., 2010, 2012 for more advanced procedures).

The directionality and intensity of sound signals are not independent features. Intensity changes with directionality, such that an increase in directionality will lead to a corresponding increase in intensity along the acoustic axis. The directivity index (DI) of the source reflects this relationship. A DI of e.g., $18 \mathrm{~dB}$ implies that sound intensity along the acoustic axis is $18 \mathrm{~dB}$ higher than it would be for an omnidirectional sound source radiating sound with the same acoustic power (Figure 5). For the piston model, the DI simply follows from the relation between size and wavelength:

$$
D I=20 \log _{10}(k \times a)
$$

where $k=2 \pi / \lambda, \lambda$ is the wavelength, and $a$ is the radius of the piston. Using measured data, the calculation of DI is slightly less simple. It requires an estimation of the sound field behind the bat and assumes that the beam is rotationally symmetric (Møhl et al., 2003).

Hence, by increasing or decreasing the directionality of the signal, the on-axis intensity inherently changes as well. M. daubentonii emits a source level of $111 \mathrm{~dB}$ SPL in the lab and $119 \mathrm{~dB}$ SPL in the field. Since the call is broader in the lab $(D I=11 \mathrm{~dB})$ than in the field $(D I=16 \mathrm{~dB})$ it follows that the bat only increases its

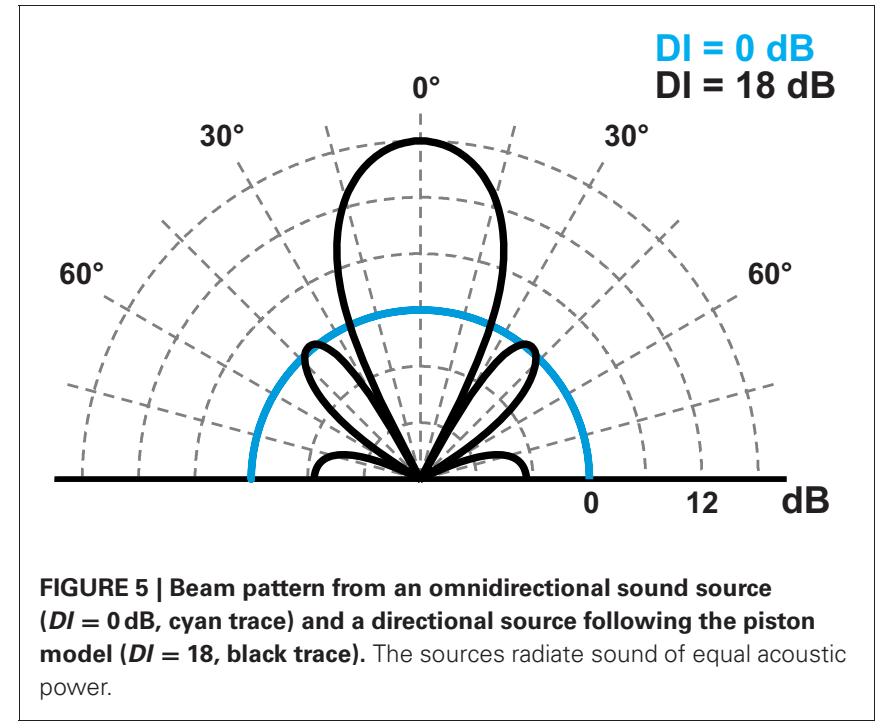

output intensity by $3 \mathrm{~dB}$ while the remaining $5 \mathrm{~dB}$ are accounted for by the greater directionality in the field (Surlykke et al., 2009).

The increase in on-axis intensity with increasing directionality also means that increasing the signal frequency does not necessarily lead to a reduction in detection distance, in spite of the increased atmospheric attenuation at higher frequencies. This is because an increase in frequency increases the signal directionality and thereby the on-axis sound level. Again the situation is simple for the piston model, where a change in frequency from $f_{1}$ to $f_{2}$ leads to a change in DI of:

$$
\Delta D I=20 \log _{10}\left(\frac{f_{2}}{f_{1}}\right)
$$

The total atmospheric attenuation depends on the distance the sound travels, whereas, an increase in on-axis sound level affects the source level and thus echo level irrespective of distance. Thus, at short echolocation ranges bats can increase frequency to achieve a higher directionality without sacrificing sonar range. At longer ranges the increase in atmospheric attenuation outweighs the increase in source level. For example a doubling in frequency from 25 to $50 \mathrm{kHz}$, increases DI and thus source level by $6 \mathrm{~dB}$ while the atmospheric attenuation increases from 0.7 to $1.7 \mathrm{~dB} / \mathrm{m}$ (at $20^{\circ} \mathrm{C}$ and $50 \%$ humidity). Thus, up to a distance of $3 \mathrm{~m}$ (two-way travel distance: $6 \mathrm{~m}$ ), the increased atmospheric attenuation at $50 \mathrm{kHz}$ does not outweigh the increase in sourcelevel. Due to the non-linear increase in atmospheric attenuation with frequency in air, the distance where these two effects cancel each other out depends on the absolute frequencies (Figure 6).

Many factors combine to define the optimal echolocation signal for a given situation. There are clear differences between bats with different feeding ecologies even when they navigate similar scenarios, indicating a critical role of feeding ecology for how evolution has shaped echolocation signals. Many phyllostomids, such as $C$. perspicillata, feed primarily on fruit and must navigate dense vegetation. Pipistrellus pygmaeus is an example of a typical vespertilionid bat hunting moving insects in open fields or along forest 


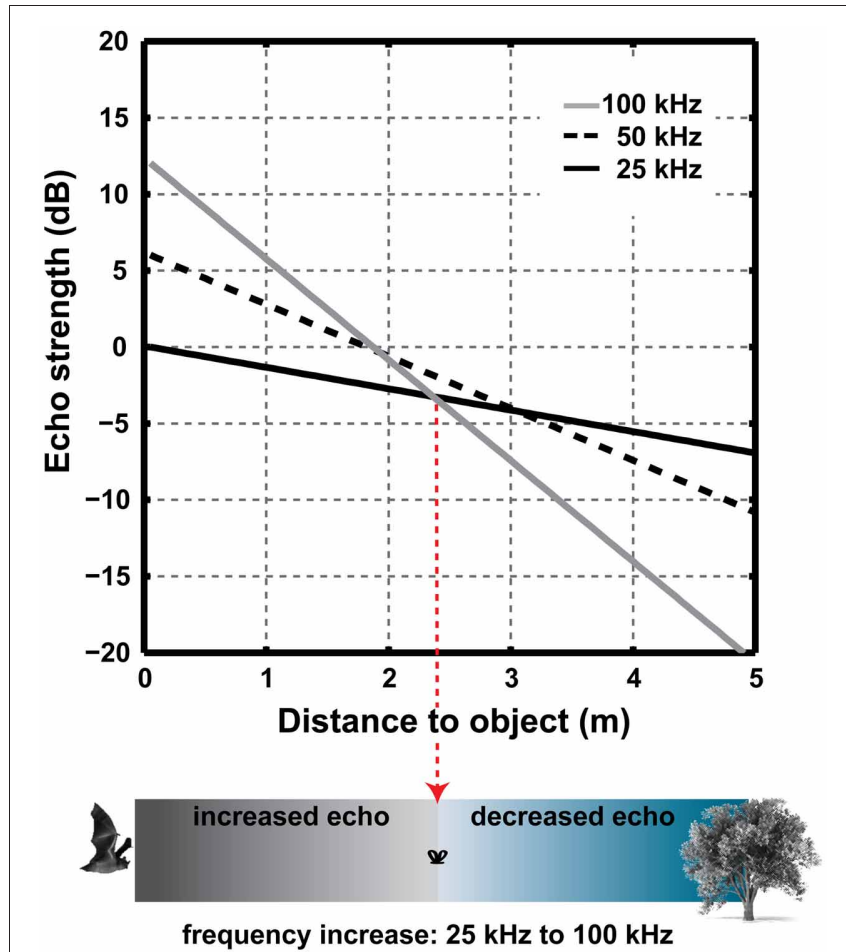

FIGURE 6 | The combined effect of increased DI and atmospheric attenuation over distance when the emitted frequency is increased from $\mathbf{2 5}$ to $\mathbf{5 0}$ to $\mathbf{1 0 0} \mathrm{kHz}$ and emitter size is constant. An increase in frequency will lead to an increase in source level and thus in echo strength at short ranges. Note that calculations do not include spherical spreading loss or scattering from the target.

edges. The requirements to the sonar systems of these two bats are very different and reflected in the combination of emitted intensity, directionality, and frequency, even when the bats are flying under similar conditions. In the lab, P. pygmaeus emits calls at $111 \mathrm{~dB}$ SPL with a DI of 12 at $55 \mathrm{kHz}$ (peak frequency). Under similar conditions, C. perspicillata emits $99 \mathrm{~dB}$ SPL, with a DI of $17 \mathrm{~dB}$ and a peak frequency of $90 \mathrm{kHz}$. Figure 7 shows the acoustic field of view for the two species and illustrates that the combined effects of intensity, directionality, and frequency generate dramatically different search volume for the two bats. The aerial hawking $P$. pygmaeus uses a sonar signal of much longer range and broader width than the gleaning C. perspicillata. P. pygmaeus searching for moving insect prey can probably "afford" to scan a relatively large volume of space with each call because its quarry will move in the foreground and thus stand out. C. perspicillata, on the other hand, must detect an inconspicuous (motionless) fruit-target in heavy clutter. By reducing the ensonified search volume it probably also focuses its attention on a smaller area and thereby increases the likelihood of detecting desirable objects caught in the sonar beam (Dukas, 2004).

\section{CONCLUSION}

The combined research on intensity and directionality of echolocation calls from bats show clear differences between restrained and unrestrained bats, and between bats flying in the lab and in

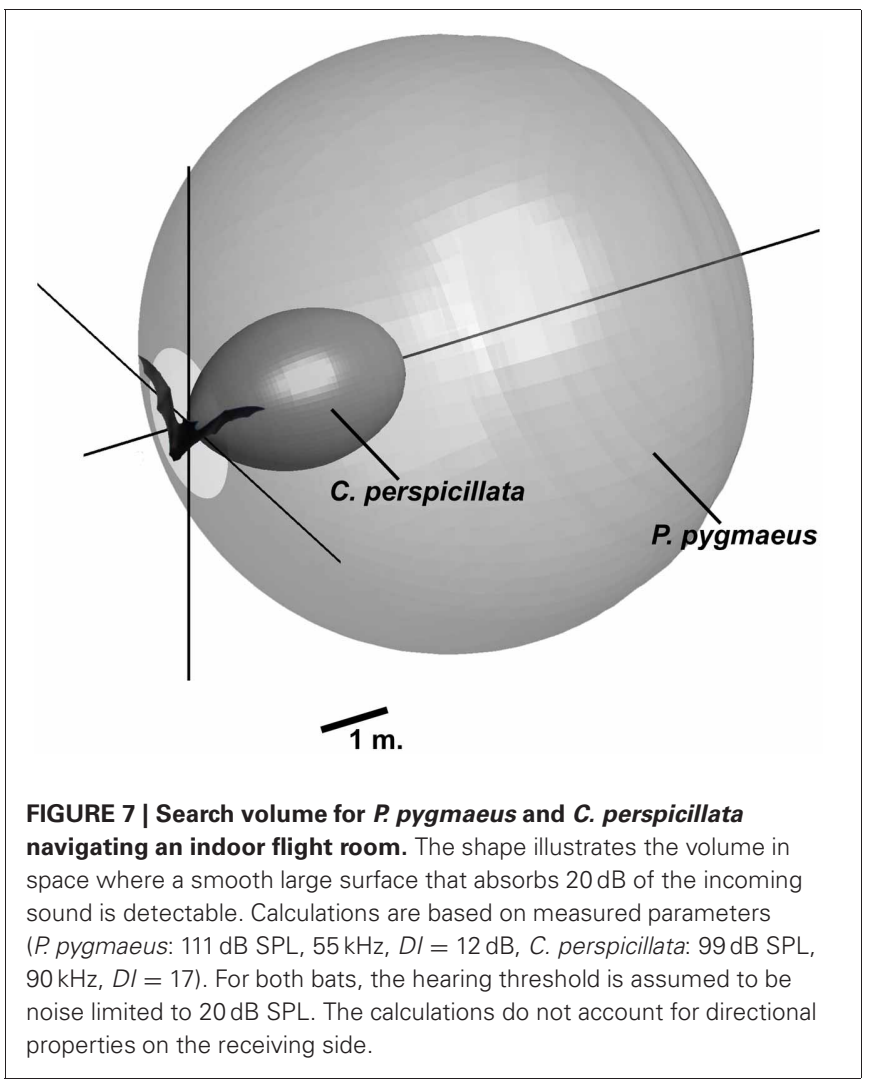

the field. These differences highlight the huge flexibility of the echolocation system and highlight the importance of active motor control for perception through echolocation. At the same time, they point to the importance of recording naturally behaving bats in the wild.

The volume of space a bat probes with its echolocation beam is a product of the emitted frequency, intensity, directionality, and call duration. The combined effect of adjustments to these components can result in dramatic changes in the overall search volume. The dynamic control of all acoustic features probably plays a key role in the flexibility and adaptability of bat echolocation and is thus a major contributor to their extreme evolutionary success across a vast range of habitats worldwide. This emphasizes the importance of determining all acoustic features, not just frequency and time parameters, to understand the function of echolocation and its adaptation through evolution to habitats and behavioral contexts.

While our knowledge of both intensity and directionality has increased substantially over recent years, it is clear that we are still barely scratching the surface. Presently, we have directionality measurements from only 17 out of more than 1000 species of echolocating bats. So what appears a general rule today may yet prove to be the exception.

\section{ACKNOWLEDGMENTS}

We thank two reviewers for their helpful comments on the manuscript. The work was funded by the Danish national research council (FNU) and the Carlsberg foundation. 


\section{REFERENCES}

ANSI. (1995). American National Standard. Method for the Calculation of the Absorption of Sound by the Atmosphere. New York, NY: Standards Secretariat. Acoustical Society of America.

Bates, M. E., Simmons, J. A., and Zorikov, T. V. (2011). Bats use echo harmonic structure to distinguish their targets from background clutter. Science 333, 627-630.

Brinkløv, S., Jakobsen, L., Ratcliffe, J. M., Kalko, E. K. V., and Surlykke, A. (2011). Echolocation call intensity and directionality in flying shorttailed fruit bats, Carollia perspicillata (Phyllostomidae). J. Acoust. Soc. Am. 129, 427-435.

Brinkløv, S., Kalko, E. K. V., and Surlykke, A. (2009). Intense echolocation calls from two 'whispering' bats, Artibeus jamaicensis and Macrophyllum macrophyllum (Phyllostomidae). J. Exp. Biol. 212, 11-20.

Brinkløv, S., Kalko, E. K. V., and Surlykke, A. (2010). Dynamic adjustment of biosonar intensity to habitat clutter in the bat Macrophyllum macrophyllum (Phyllostomidae). Behav. Ecol. Sociobiol. 64, 1867-1874.

Dukas, R. (2004). Causes and consequences of limited attention. Brain Behav. Evol. 63, 197-210.

Esser, K.-H., and Daucher, A. (1996). Hearing in the FM-bat Phyllostomus discolor: a behavioral audiogram. J. Comp. Physiol. A 178, 779-785.

Faure, P. A., and Barclay, R. M. R. (1994). Substrate-gleaning versus aerial-hawking: plasticity in the foraging and echolocation behavior of the long-eared bat, Myotis evotis. J. Comp. Physiol. A 174, 651-660.

Feng, L., Gao, L., Lu, H., and Müller, R. (2012). Noseleaf dynamics during pulse emission in horseshoe bats. PLoS ONE 7:e34685. doi: 10.1371/journal.pone.0034685

Geipel, I., Jung, K., and Kalko, E. K. (2013). Perception of silent and motionless prey on vegetation by echolocation in the gleaning bat Micronycteris microtis. Proc. Biol. Sci. 280, 20122830.

Ghose, K., and Moss, C. F. (2003). The sonar beam pattern of a flying bat as it tracks tethered insects. J. Acoust. Soc. Am. 114, 1120-1131.

Goerlitz, H. R., Ter Hofstede, H. M., Zeale, M. R. K., Jones, G., and Holderied, M. W. (2010). An aerialhawking bat uses stealth echolocation to counter moth hearing. Curr. Biol. 20, 1568-1572.

Griffin, D. R. (1958). Listening in the Dark. New York, NY: Yale
University Press (2nd Edn. 1986, Cornell University).

Griffin, D. R., Webster, F. A., and Michael, C. R. (1960). The echolocation of flying insects by bats. Anim. Behav. 8, 141-154.

Hartley, D. J. (1992a). Stabilization of perceived echo amplitudes in echolocating bats. I. Echo detection and automatic gain control in the big brown bat, Eptesicus fuscus, and the fishing bat, Noctilio leporinus. J. Acoust. Soc. Am. 91, 1120-1132.

Hartley, D. J. (1992b). Stabilization of perceived echo amplitudes in echolocating bats. II. The acoustic behavior of the big brown bat, Eptesicus fuscus, when tracking moving prey. J. Acoust. Soc. Am. 91, 1133-1149.

Hartley, D. J., Campbell, K. A., and Suthers, R. A. (1989). The acoustic behavior of the fish-catching bat, Noctilio leporinus, during prey capture. J. Acoust. Soc. Am. 86, 8-27.

Hartley, D. J., and Suthers, R. A. (1987). The sound emission pattern and the acoustical role of the noseleaf in the echolocating bat, Carollia perspicillata. J. Acoust. Soc. Am. 82, 1892-1900.

Hartley, D. J., and Suthers, R. A. (1989). The sound emission pattern of the echolocating bat, Eptesicus fuscus. J. Acoust. Soc. Am. 85, 1348-1351.

Henson, O. W. (1965). The activity and function of the middle-ear muscles in echo-locating bats. J. Physiol. 180, 871-887.

Henze, D., and O’Neill, W. E. (1991). The emission pattern of vocalizations and directionality of the sonar system in the echolocating bat, Pteronotus parnelli. J. Acoust. Soc. Am. 89, 2430-2434.

Hiryu, S., Hagino, T., Riquimaroux, H., and Watanabe, Y. (2007). Echointensity compensation in echolocating bats (Pipistrellus abramus) during flight measured by a telemetry microphone. J. Acoust. Soc. Am. 121, 1749-1757.

Hiryu, S., Katsura, K., Lin, L.-K., Riquimaroux, H., and Watanabe, Y. (2006). Radiation pattern of echolocation pulse in Taiwanese leaf-nosed bat, Hipposideros terasensis. Acoust. Sci. Tech. 27, 108-110.

Hiryu, S., Shiori, Y., Hosokawa, T., Riquimaroux, H., and Watanabe, Y. (2008). On-board telemetry of emitted sounds from free-flying bats: compensation for velocity and distance stabilizes echo frequency and amplitude. J. Comp. Physiol. A Neuroethol. Sens. Neural Behav. Physiol. 194, 841-851.

Holderied, M. W., Korine, C., Fenton, M. B., Parsons, S., Robson, S., and
Jones, G. (2005). Echolocation call intensity in the aerial hawking bat Eptesicus bottae (Vespertilionidae) studied using stereo videogrammetry. J. Exp. Biol. 208, 1321-1327.

Ibáñez, C., Juste, J., López-Wilchis, R., and Albuja, L., Núñez-Garduño, A. (2002). Echolocation of three species of sac-winged bats (Balantiopteryx). J. Mamm. 83, 1049-1057.

Jakobsen, L., Kalko, E., and Surlykke, A. (2012). Echolocation beam shape in emballonurid bats, Saccopteryx bilineata and Cormura brevirostris. Behav. Ecol. Sociobiol. 66, 1493-1502.

Jakobsen, L., Ratcliffe, J. M., and Surlykke, A. (2013). Convergent acoustic field of view in echolocating bats. Nature 493, 93-96.

Jakobsen, L., and Surlykke, A. (2010). Vespertilionid bats control the width of their biosonar sound beam dynamically during prey pursuit. Proc. Natl. Acad. Sci. U.S.A. 107 13930-13935.

Jones, G. (1999). Scaling of echolocation call parameters in bats. J. Exp. Biol. 202, 3359-3367.

Kalko, E. K. V., and Schnitzler, H.-U. (1989). The echolocation and hunting behavior of Daubenton's bat, Myotis daubentoni. Behav. Ecol. Sociobiol. 24, 225-238.

Kalko, E. K. V., and Schnitzler, H.-U. (1993). Plasticity in echolocation signals of European pipistrelle bats in search flight: implications for habitat use and prey detection. Behav. Ecol. Sociobiol. 33, 415-428.

Koay, G., Heffner, H. E., and Heffner, R. S. (1997). Audiogram of the big brown bat (Eptesicus fuscus). Hear Res. 105, 202-210.

Koblitz, J. C., Stilz, P., Pflasterer W., Melcon, M. L., and Schnitzler, H.-U. (2011). Source level reduction and sonar beam aiming in landing big brown bats (Eptesicus fuscus). J. Acoust. Soc. Am. 130, 3090-3099.

Koblitz, J. C., Stilz, P., and Schnitzler H.-U. (2010). Source levels of echolocation signals vary in correlation with wingbeat cycle in landing big brown bats (Eptesicus fuscus). J. Exp. Biol. 213, 3263-3268.

Lawrence, B. D., and Simmons, J. A. (1982). Measurements of atmospheric attenuation at ultrasonic frequencies and the significance for echolocation by bats. J. Acoust. Soc. Am. 71, 585-590.

Matsuta, N., Hiryu, S., Fujioka, E. Yamada, Y., Riquimaroux, H., and Watanabe, Y. (2013). Adaptive beam-width control of echolocation sounds by CF-FM bats, Rhinolophus ferrumequinum nippon, during prey-capture flight. J. Exp. Biol. 216, 1210-1218.

Miller, L. A., and Surlykke, A. (2001). How some insects detect and avoid being eaten by bats: tactics and countertactics of prey and predator. Bioscience 51, 571-582.

Mogensen, F., and Møhl, B. (1979). Sound radiation patterns in the frequency domain of cries from a Vespertilionid bat. J. Comp. Physiol. A 134, 165-171.

Møhl, B. (1988). "Target detection by echolocating bats", in Animal Sonar, eds P. E. Nachtigall and P. W. B. Moore (New York, NY: Plenum), 435-450.

Møhl, B., Wahlberg, M., Madsen, P. T., Heerfordt, A., and Lund, A. (2003). The monopulsed nature of sperm whale clicks. J. Acoust. Soc. Am. 114 1143-1154.

Möhres, F. P., and Neuweiler, G. (1966). Die Ultraschallorientierung der Grossblatt-Fledermäuse (Chiroptera - Megadermatidae). JCP 53, 195-227.

Nørum, U., Brinkløv, S., and Surlykke, A. (2012). New model for gain control of signal intensity to object distance in echolocating bats. J. Exp. Biol. 215, 3045-3054.

Pye, J. D. (1993). Is fidelity futile? The 'true' signal is illusory, especially with ultrasound. Bioacoustics 4, 271-286.

Ratcliffe, J. M., Elemans, C. P. H., Jakobsen, L., and Surlykke, A. (2013). How the bat got it buzz. Biol. Lett. 9:20121031. doi: 10.1098/rsbl.2012.1031

Schmidt, S., Türke, B., and Vogler, B. (1992). Behavioural audiogram from the bat, Megaderma lyra (Geoffroy, 1810; Microchiroptera). Myotis 21-22, 62-66.

Schnitzler, H.-U., and Grinnell, A. D. (1977). Directional sensitivity of echolocation in the horseshoe bat, Rhinolophus ferrumequinum. I Directionality of sound emission. J. Comp. Physiol. A 116, 51-61.

Schnitzler, H.-U., and Kalko, E. K. V. (2001). Echolocation by insecteating bats. Bioscience 51, 557-569.

Shimozawa, T., Suga, N., Hendler, P. and Schuetze, S. (1974). Directional sensitivity of echolocation system in bats producing frequencymodulated signals. J. Exp. Biol. 60, 53-69.

Simmons, J. A. (1969). Acoustic radiation patterns for the echolocating bats Chilonycteris rubiginosa and Eptesicus fuscus. J. Acoust. Soc. Am. 46, 1054-1056.

Stamper, S. A., Bates, M. E., Benedicto, D., and Simmons, J. A. (2009) Role of broadcast harmonics in 
echo delay perception by big brown bats. J. Comp. Physiol. A Neuroethol. Sens. Neural Behav. Physiol. 195, 79-89.

Strother, G. K., and Mogus, M. (1970). Acoustical beam patterns for bats: some theoretical considerations. J. Acoust. Soc. Am. 48, 1430-1432.

Suga, N., and Jen, P. H.-S. (1975). Peripheral control of acoustic signals in the auditory system of echolocating bats. J. Exp. Biol. 62, 277-311.

Surlykke, A. (1988). "Interaction between echolocating bats and their prey," in Animal Sonar. Processes and Performance, eds P. E. Nachtigall and P. W. B. Moore (New York, London: Plenum Press), 551-566.

Surlykke, A., Filskov, M., Fullard, J. H., and Forrest, E. (1999). Auditory relationships to size in noctuid moths: bigger is better. Naturwissenschaften 86, 238-241.
Surlykke, A., and Kalko, E. K. V. (2008). Echolocating bats cry out loud to detect their prey. PLoS ONE 3:e2036. doi: 10.1371/journal. pone. 0002036

Surlykke, A., Miller, L. A., Møhl, B., Andersen, B. B., ChristensenDalsgaard, J., and Jørgensen, M. B. (1993). Echolocation in two very small bats from Thailand: Craseonycteris thonglongyai and Myotis siligorensis. Behav. Ecol. Sociobiol. 33, 1-12.

Surlykke, A., Pedersen, S. B., and Jakobsen, L. (2009). Echolocating bats emit a highly directional sonar sound beam in the field. Proc. Biol. Sci. 276, 853-860.

Urick, R. J. (1983). Principles of Underwater Sound. New York, NY: Peninsula Publishing.

Vanderelst, D., De Mey, F., Peremans, H., Geipel, I., Kalko, E. K. V., and Firzlaff, U. (2010). What noseleaves do for fm bats depends on their degree of sensorial specialization.
PLoS ONE 5:e11893. doi: 10.1371/ journal.pone.0011893

Vanderelst, D., Jonas, R., and Herbert, P. (2012). The furrows of Rhinolophidae revisited. J. R. Soc. Interface 9, 1100-1103.

Wenstrup, J. J. (1984). Auditory sensitivity in the fish-catching bat, Noctilio leporinus. J. Comp. Physiol. A 155, 91-101.

Zhuang, Q., and Müller, R. (2006) Noseleaf furrows in a horseshoe bat act as resonance cavities shaping the biosonar beam. Phys. Rev. Lett. 97, 218701-218704.

Zhuang, Q., and Müller, R. (2007). Numerical study of the effect of the noseleaf on biosonar beamforming in a horseshoe bat. Phys. Rev. E 76:051902. doi: 10.1103/physreve. 76.051902

Conflict of Interest Statement: The authors declare that the research was conducted in the absence of any commercial or financial relationships that could be construed as a potential conflict of interest.

Received: 19 February 2013; accepted: 05 April 2013; published online: 25 April 2013.

Citation: Jakobsen L, Brinkløv $S$ and Surlykke A (2013) Intensity and directionality of bat echolocation signals. Front. Physiol. 4:89. doi: 10.3389/fphys. 2013.00089

This article was submitted to Frontiers in Integrative Physiology, a specialty of Frontiers in Physiology.

Copyright (C) 2013 Jakobsen, Brinkløv and Surlykke. This is an open-access article distributed under the terms of the Creative Commons Attribution License, which permits use, distribution and reproduction in other forums, provided the original authors and source are credited and subject to any copyright notices concerning any third-party graphics etc. 